\title{
Influence of the operational point in the photovoltaic converters performance
}

\author{
César Tablero-Crespo
}

\begin{abstract}
We evaluate the constrained efficiently of the coupling between a photovoltaic device and a device supplying a constant voltage and/or current. The former can be used with an electrochemical device to store solar to chemical energy. Single- and multi-gap semiconductors are used to evaluate the photovoltaic devices. Unlike other approaches, no approximation is made to fix the output voltage and/or current and the method is not limited to non-degenerate semiconductors. The problem is solved globally with a generalized methodology that allows for the analysis of any type of absorption coefficients and solar spectra.
\end{abstract}

Keywords: photovoltaic, Solar Fuel, efficiency

\section{Introduction}

A photovoltaic device (PVD) absorbs photons from sunlight transforming their energy into photo-voltage and photo-current. The energy can be used directly or to supply other devices (OD), for example to convert this solar energy into chemical energy. Batteries and fuel cells (FCs) are two examples of solar energy storage devices converted to chemical energy. Later these devices convert the stored chemical energy into electrical energy. In order to carry out the conversion to fuels, the PVD directly coupled to the electrochemical cell must provide the photo-voltage for the half-cell potentials of the redox reaction $(\sim 1.23 \mathrm{eV}$ for water splitting $[1,2])$. This photo-voltage is the difference between the electrochemical potential of electrons and holes under illumination. Because of the losses due to anodic and cathodic overvoltages, resistances, etc, the resulting photo-voltage $\mathrm{V}>1.6-1.9 \mathrm{eV}$ [2]. Therefore when the PVD is coupled the photo-voltage is constrained. Thus many PVDs with high solar efficiencies are not able to provide sufficient voltage to split water into $\mathrm{O}_{2}$ and $\mathrm{H}_{2}$ gases, or to reduce $\mathrm{CO}_{2}$.

The direct electrical connection between a PVD and OD is equivalent to constraining the currents and voltages of the PVD and $O D$ to be identical (figure 1). If the PVD and the OD have the characteristics current-voltage $F_{P V D}(J, V)=0$ and $F_{O D}(J, V)=0$, the operational point $\left(J_{o}, V_{o}\right)$ must satisfy the two characteristics equations simultaneously. Graphically, this constraint identifies the operational point of the coupled PVD-OD device as the point at which the current-voltage properties of the PVD and of the OD intersect. This mode of operation generally results in the PVD producing less than its maximum output and efficiency, i.e. there is a coupling loss (figure 1).

Alternatives to direct coupling are also possible. For example, power electronics could convert the electrical output of one or more solar cells to the required electrical input of the electrochemistry cell. Practically, the choice of a coupling scheme would be dictated by matching the tolerable complexity, reliability, and cost of an application with the available coupling solutions. For example inverters have a $90 \%-$ $98 \%$ efficiency conversion but inverter efficiency depends on the inverter load. The aforementioned efficiency conversion is the maximum efficiency peak. Below $10 \%-15 \%$ of power output, the efficiency is quite low whereas at high output power, the efficiency is steadily high. Therefore power electronics solutions are best suited for use at high output power, for example with solar modules, but not for solar cells with low output power where the inverters are very inefficient.

The efficiency $\eta$ with which PVD power is transferred to the $\mathrm{OD}$ is $\eta=J_{0} V_{o} / P_{\text {inc }}$ where $P_{\text {inc }}$ is the irradiance of the incident (sun light) spectrum. As we have described, this efficiency is lower than the maximum efficiency $\eta_{m}$ of the 

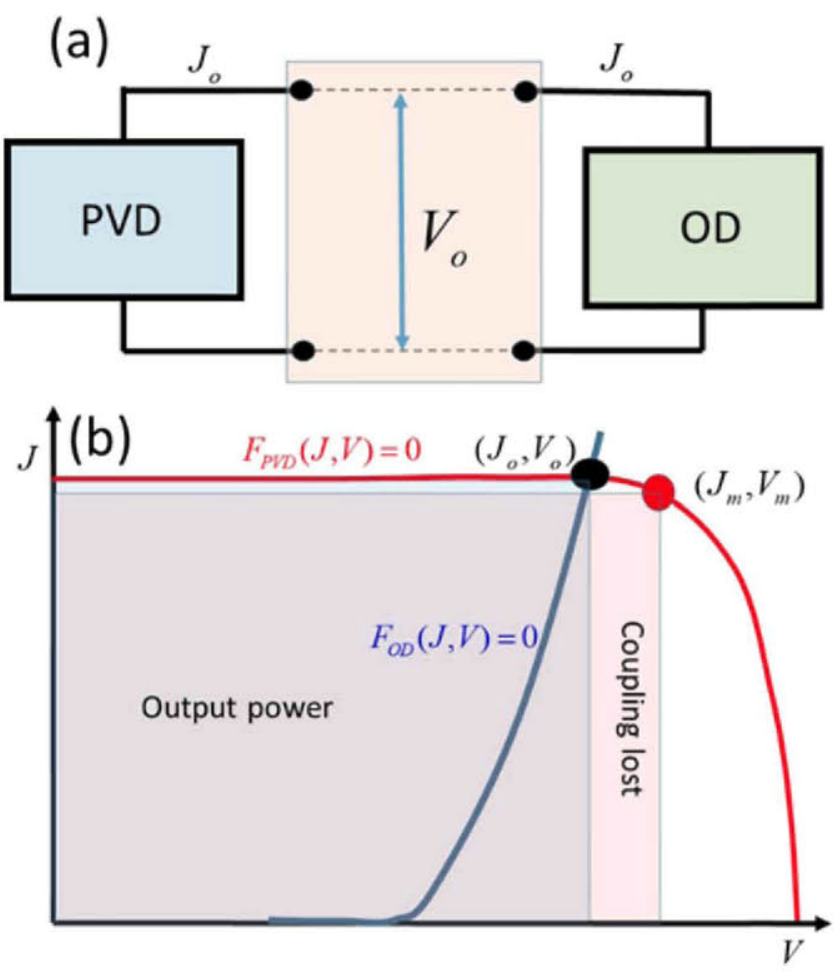

Figure 1. (a) Block diagram of the PVD-OD coupling. (b) The current density-voltage $(J-V)$ characteristic of the coupled PVD-OD device where $F_{P V D}(J, V)=0$ and $F_{O D}(J, V)=0$ are the characteristic of the PVD and OD without coupling. The point $\left(J_{o}, V_{o}\right)$ identifies the operational point, and $\left(J_{m}, V_{m}\right)$ the maximum power point of the PVD.

PVD because the operational point $\left(J_{o}, V_{o}\right)$, in general, is different to the maximum power point $\left(J_{m}, V_{m}\right)$. Therefore $J_{o} V_{o} \leqslant J_{m} V_{m}$ and $\eta \leqslant \eta_{m}$. It produces a coupling loss (figure 1).

Note that $\eta$ already takes into account the coupling efficiency because it is obtained with the restriction of the $V_{o}$ and/or the $J_{o}$ constant. For example if the OD is a electrochemical cell the constant operational voltage will be the sum of the redox potential, $V_{\text {redox }}$, to carry out the fuel production and the voltage losses, $V_{\text {lost }}$, because of the electrode overpotentials and other causes, i.e. $V_{o}=V_{\text {redox }}+V_{\text {lost }}$. The overall efficiency of the two devices connected in series is $\eta_{A}=\eta \cdot \eta_{O D}$, where $\eta_{O D}$ is the efficiency at the operating point of the OD connected to the PVD. For example, for a fuel-cell, the power stored as chemical energy is $J_{o} V_{\text {redox }}$ while the power supplied from the PVD is $J_{o} V_{o}$. Therefore $\eta_{O D}=V_{\text {redox }} / V_{o}$. Note that $\eta_{A}$ is the theoretical efficiency which can be reached with semiconductor-based electrochemical devices for the conversion of solar light into storable redox energy [3, 4]. Assuming that the losses are $V_{\text {loss }}$, the energy storage efficiency is $\eta_{\text {stor }}=J_{o} V_{\text {redox }} / P_{\text {inc }}$, which can be easily checked as $\eta_{\text {stor }} \equiv \eta_{A}=\eta \cdot \eta_{O D}$. Therefore the results obtained with the restriction of the $V_{o}$ constant are immediately transferred to energy storage efficiency multiplying $\eta$ by $\eta_{O D}=$ $V_{\text {redox }} / V_{0}$

Conversion maximum efficiencies when the energy is stored in a chemical fuel have already been derived basing on the theory developed by Bolton [5]. This theory has been applied mainly to the conversion efficiencies for photo-electrochemical $\mathrm{H}_{2} \mathrm{O}$ splitting for different overvoltages [3, 4]. In these applications only single-gap semiconductors have been evaluated. As well as using an equivalent circuit analysis, the efficiency of a series-connected string of identical single-gap solar cells coupled to an electrochemical process that produces storable fuels has been described previously [6].

Many single-junction solar cells do not generate enough voltage to store the energy in a chemical fuel. To overcome this limitation multi-junction configurations are used. If $N$ identical single-gap solar cells are wired in series, their voltages are approximately additive $\left(V_{N} \sim N V_{1}\right)$, where the subindex indicates the number of solar cells. It avoids the voltage problem: increasing the number of solar cells increases the output voltage. However the current density of the entire system will decrease as $J_{N} \sim J_{1} / N$. Of course, the efficiency is similar to the single-gap solar cell. Nevertheless, if the voltage is constrained to the operational voltage $V_{o}$, the only way to increase the efficiency is to increase the current. Therefore, this approach avoids the voltage problem but it does not increase the energy storage efficiency.

From the previous discussion the energy storage efficiency can be enlarged by increasing the current $J_{o}$. An option presented in this work is to use multi-gap semiconductors with intermediate bands (IBs) [7-9] between the valence band (VB) and the conduction band (CB) of the host single-gap semiconductor. With these multi-gap semiconductors the photons of lower energy than the host semiconductor gap can be exploited. These photons would not be absorbed by a single-gap semiconductor. In this way the current and the efficiency increases with respect to single-gap semiconductors even though the voltage and current are restricted, as it will be shown later.

The main contributions and novelties of this work, which will be discussed below, are: (i) In addition to evaluating the conventional single-gap semiconductor we also analyze multigap semiconductors; (ii) no approximations are made to fix the output voltage and/or current; (iii) the emission current due to the radiative recombination is considered, and the Fermi-Dirac and Bose-Einstein statistics are not approximated by the Maxwell-Boltzmann statistic, i.e. the treatment is not limited to non-degenerate semiconductors. The problem is approached using constrained non-lineal optimization algorithms.

\section{Methodology}

\subsection{Characteristic $\mathrm{J}-\mathrm{V}$ of single- and multi-gap solar cell}

Single-gap semiconductors have two bands, the VB and the $\mathrm{CB}$, and only one gap. Photon absorption causes $\mathrm{V} \rightarrow \mathrm{C}$ transitions. The photon absorption effectiveness depends on the absorption coefficient $\alpha_{V C}$. Generalizing for a multi-gap semiconductor where several bands are possible we state this as $\mathrm{A} \rightarrow \mathrm{B}$ transitions with the absorption coefficient $\alpha_{A B}$, in which the total absorption coefficient is $\alpha_{T}=\sum_{A} \sum_{B>A} \alpha_{A B}$. In this generalization, single-gap semiconductors are then a particular case with only two bands. 
The photon absorption from $\mathrm{A}$ to $\mathrm{B}$ bands generates a photocurrent $J_{A B}$. Therefore the net current for the $\mathrm{A}$ band is $J_{A}=\sum_{B \neq A} J_{B A}$, with $J_{A B}=-J_{B A}$. Supposing a quantum efficiency equal to one, this photocurrent is the difference between the absorbed and emitted photons [7-10] transformed to current: $J_{A B}=J_{A B}^{(a)}-J_{A B}^{(e)}$, where $J_{A B}^{(\kappa)}=$ $q \int a\left(E, \alpha_{T}, w\right)\left(\alpha_{A B} / \alpha_{T}\right) \Phi_{\kappa}^{(A B)}(E) d E$ ( $\kappa=a, e$ for absorption and emission respectively). In this expression $a\left(E, \alpha_{T}, w\right) \simeq 1-e^{-\alpha_{T} w}$ is the absorptivity, $w$ the thickness of the absorbent material, $q$ is the electron charge, and $\Phi_{a}^{(A B)}$ and $\Phi_{e}^{(A B)}$ are the incident and the emitted spectrum photon flux density respectively for the $\mathrm{A} \rightarrow \mathrm{B}$ transitions. For the incident sunlight spectrum $\left(\Phi_{a}^{(A B)} \equiv \Phi_{a}\right)$ we have used the AM1.5G [11] spectrum. For the emitted spectrum we have used the conventional approach considering that the device emits radiation as a black-body at $\mathrm{T}=300 \mathrm{~K}$ [7-10]. With this assumption $\Phi_{e}^{(A B)}=\left(2 \pi / h^{3} c^{2}\right) E^{2}\left(\alpha_{A B} / \alpha_{T}\right)$ $\sum_{P} \sum_{Q>P}\left(\alpha_{P Q} / \alpha_{T}\right) b_{P Q}\left(E, T, \mu_{Q P}\right)$, where $b_{B A}\left(E, T, \mu_{B A}\right)=$ $\left[e^{\left(E-\mu_{B A}\right) / k T}-1\right]^{-1}$ is the Bose-Einstein factor, $k$ is the Boltzmann constant, $h$ the Planck constant, $c$ the speed of light in vacuum, and $\mu_{B A}=\mu_{B}-\mu_{A}$ is the chemical potential associated with the radiation emitted between the $\mathrm{A}$ and $\mathrm{B}$ bands [7-10]). Note that the emission current, via the Boltzmann factors $b_{B A} \equiv b_{B A}\left(E, T, \mu_{B A}\right)=\left[e^{\left(E-\mu_{B A}\right) / k T}-1\right]^{-1}$, depends on the variables $\mu_{A}$ of the multi-variable non-linear problem.

For a two-terminal multi-gap semiconductor the current $J$ is taken from the VB (hole current $J_{V}$ ) and CB (electron current $\left.-J_{C}\right)$. Therefore $J=J_{V}=-J_{C}$. No current is extracted from the IBs, i.e. $J_{I}=0(I \neq V, C)$. The voltage $V$ between terminals is $V=\mu_{C}-\mu_{V}$, i.e. the difference between the $\mathrm{CB}$ and VB chemical potentials. Therefore, to obtain the $J-V$ relationship it is necessary to solve simultaneously a multi-variable non-linear problem where the set of equations are $J=J_{V}=-J_{C}, J_{I}=0(I \neq V, C)$, which must be solved as a function of the chemical potentials $\mu_{A}$.

\subsection{Multi-gap solar cell efficiency with restricted $J$ or $V$}

The coupling PVD-OD involves restricting the voltage $V$ and/or the current $J$ to $V_{0}$ or/and $J_{0}$, where $V_{0}$ and $J_{0}$ are the operational voltage and current respectively (figure 1). Therefore mathematically the problem is to maximize the PVD efficiency constricting the voltage and/or current to the operational point, i.e. a constrained multi-variable non-linear optimization problem. This methodology has been used previously in several author publications using the habitual step [12] and from first-principles absorption coefficients [13-16].

\section{Results and discussion}

Using the methodology described in the previous section we have evaluated the efficiencies of the PVD for several situations considering: (i) the output voltage constrained to $V_{0}$; (ii) the output current restricted to $J_{0}$.
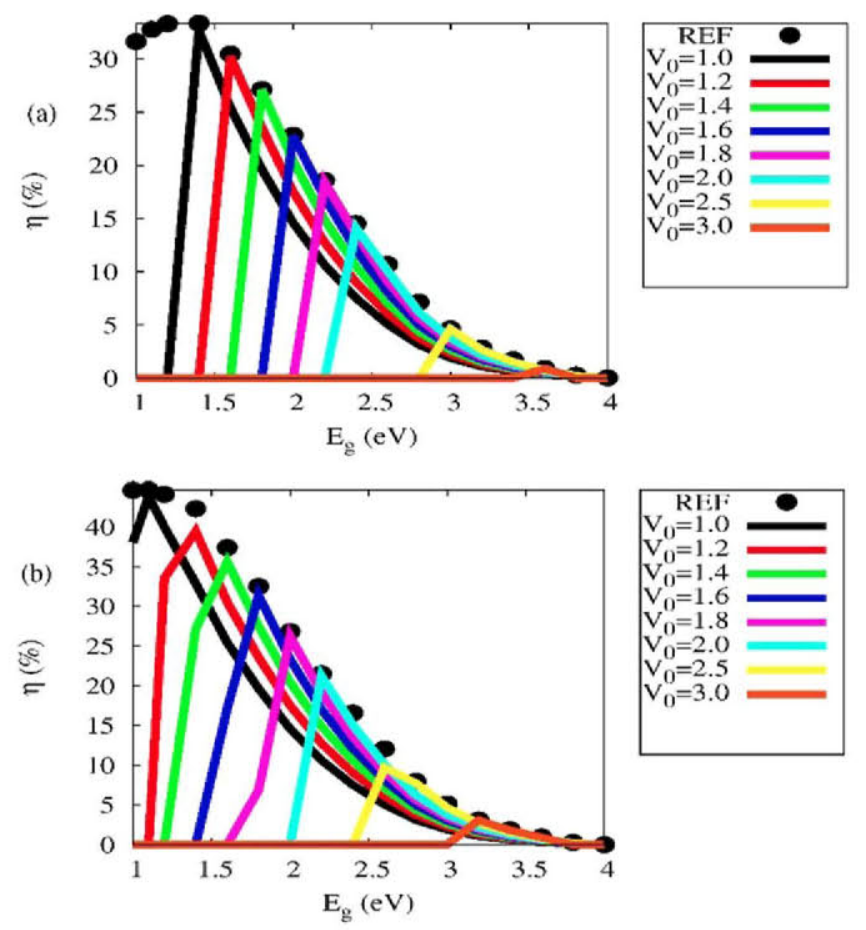

Figure 2. Maximum efficiency of a 2-band single-gap semiconductor as an energy gap energy function for several output photo-voltage $\mathrm{V}_{\mathrm{O}}(\mathrm{V})$ values using standard conditions (AM $1.5 \mathrm{G}, 1 \mathrm{~kW} \mathrm{~m}^{-2}$,

$\left.25^{\circ} \mathrm{C}\right)$ and (a) without sunlight concentration $(\mathrm{X}=1)$, and (b) maximum concentration $X=\max =46200$. The curve labeled as 'REF' corresponds to the overall maximum efficiency, i.e. without restricting the current or voltage to a fixed value.

\subsection{Device with a fixed voltage}

The maximum efficiency obtained for a conventional singlegap semiconductor when the output voltage $V_{0}$ is fixed to several values is shown in figure 2. Obviously, the PVD can only be operated with a semiconductor having a wide enough gap in order to provide the necessary voltage. The same applies to a storage cell. As it has been discussed previously these results are directly transferred to the energy storage efficiency multiplying $\eta$ by $\eta_{O D}=V_{\text {redox }} / V_{o}$.

When the output voltage $V_{0}$ is fixed the efficiency increases with the photocurrent $J$. In principle, there exist various possibilities to improve the efficiency. One such option is to increase the sunlight concentration to utilize two threshold absorbers with different gaps in series, etc. Another option presented in this work is to use a multi-gap semiconductor [7-9] with intermediate (I) bands between the valence (V) and conduction (C) bands of the conventional single-gap semiconductor. For a single-gap semiconductor the current comes from the $\mathrm{V} \rightarrow \mathrm{C}$ transitions. For a multigap semiconductor, in addition to the $\mathrm{V} \rightarrow \mathrm{C}$ transitions, there are $\mathrm{V} \rightarrow \mathrm{I} \rightarrow \mathrm{C}$ transitions generating extra current, and thus increasing the efficiency. Then photons with less energy than the V-C gap can be used to generate more photo-current and to increase the efficiency. It can be observed by comparing the efficiencies in figures 2 and 3 for single- and double-gap semiconductors respectively. 

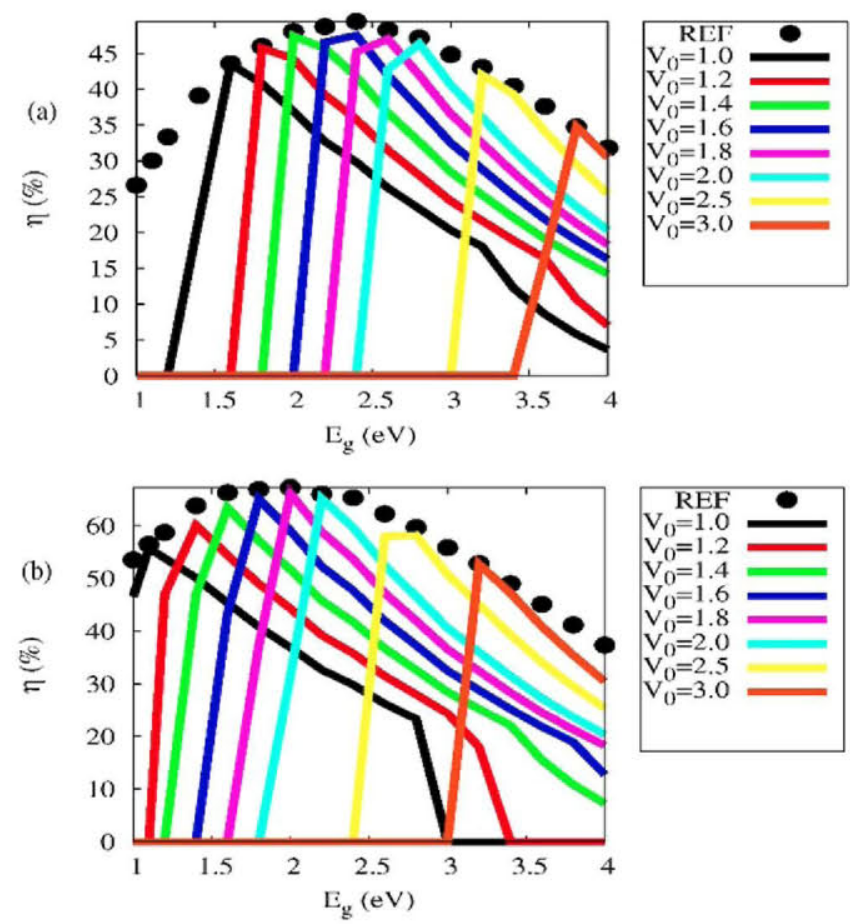

Figure 3. The same legend as that in figure 2, but for a double-gap (3-band) semiconductor.

\subsection{Energy storage efficiency}

In order to contrast our results with previous results in the literature we have compared our results with the results obtained with Bolton's approach [5]. This method has been used for photoelectron-chemical fuel conversion with different overvoltages [3, 4]. The main approximation in this approach are: (i) a photochemical converter is considered to be an ideal chemical system obeying Boltzmann statistics. It implied that the solar converter is non-degenerate; (ii) the current generated from photon absorption is much greater than the black-body induced emission current. According to this approach, the energy storage efficiency to voltage $V_{\text {redox }}$ is $[4,5] \eta_{A}^{(B)}=J_{a}\left(1-e^{-q\left(V-V_{0}\right) / k T}\right) \cdot\left(q V_{\text {redox }}\right) / P_{\text {inc }}$ if $V \geqslant V_{o}$, and zero otherwise. In this expression $V_{o}=V_{\text {redox }}+V_{\text {loss }}$ is the operating voltage and $q V_{\text {redox }}$ is the stored energy power.

Figure 4 shows the energy storage efficiency $\eta_{A}$ for the photo-electrochemical $\mathrm{H}_{2} \mathrm{O}$ splitting as a function of gap for different losses $V_{\text {lost }}$. It has been assumed that storage energy is $q V_{\text {redox }}=1.23 \mathrm{eV}$ (water splitting), and an $n$-type electrode combined with a metal counter electrode. The losses could be considered as losses due to overpotentials and/or other causes. The conversion efficiency (figure 4(a)), when the operating voltage is $V_{0}=V_{\text {redox }}+V_{\text {lost }}$ (and the stored energy $q V_{\text {redox }}$ ), is slightly larger near the maxima than that obtained with Bolton's approach. The main difference is that with Bolton's approach the separation of the pseudo-Fermi energies of the photovoltaic converter is larger than or equal to $V_{0}$ (figure $4(\mathrm{~b})$ ), whereas with the methodology used in this work this separation is equal to the operating voltage $V_{0}$. In summary, the operating voltage with Bolton's approach is restricted to $V_{0} \geqslant V_{\text {redox }}+V_{\text {lost }}$, whereas
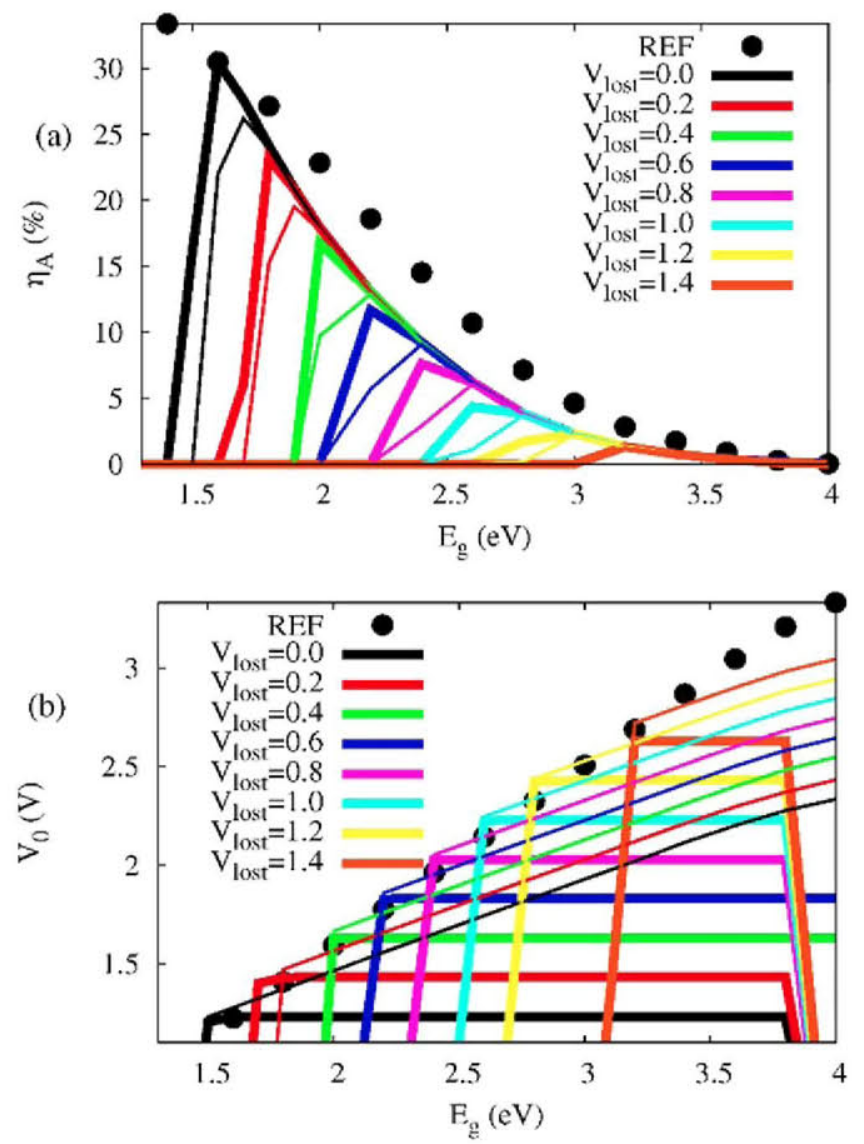

Figure 4. (a) Maximum efficiency $\eta_{A}$ into storable redox energy and (b) operating voltage of a 2-band single-gap semiconductor as an energy gap energy function for different voltage losses $V_{\text {lost }}$. The assumed storage energy is $q V_{\text {redox }}=1.23 \mathrm{eV}$. Standard conditions (AM1.5 G, $1 \mathrm{~kW} \mathrm{~m}{ }^{-2}, 25^{\circ} \mathrm{C}$ ) without sunlight concentration $(X=1)$ have also been assumed. The curves with thick and thin lines correspond to the results of this work and those obtained using the Bolton's approach [5] respectively. The dot curve labeled as 'REF' corresponds to the overall maximum efficiency, i.e. without restricting the current or voltage to a fixed value.

with the method used in this work the operating voltage is restricted to $V_{0}=V_{\text {redox }}+V_{\text {lost }}$.

Figure 5 is similar to figure 4(a), but for a double-gap (3band) semiconductor. Comparing the two figures, the storable energy efficiencies with a double-gap semiconductor are larger than those with a single-gap semiconductor. The reason is that the former are able to generate more current for the same voltage.

In both single- and multi-gap solar cells the efficiencies decrease with increasing $V_{\text {lost }}$. The efficiencies for $V_{\text {lost }}>0$ are always less than or equal to those for $V_{\text {lost }}=0$. The maximum theoretical efficiencies obtained with Bolton's approach are $\sim 27 \%$ and $\sim 42 \%$ for $V_{\text {lost }}=0$ with single- and doublejunction semiconductors. With the methodology used in this work the absolute maximums of storable energy efficiencies are $\sim 30.5 \%$ and $\sim 47.4 \%$ for $V_{\text {lost }}=0$ with single- and double-gap semiconductor. The efficiency of the double-gap is greater than the double-junction semiconductors. 


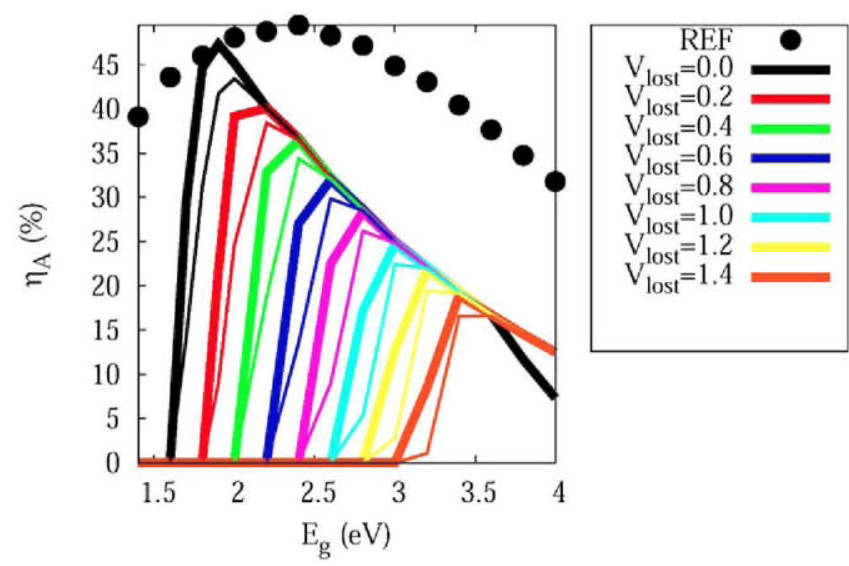

Figure 5. Same legend as that in figure 4(a), but for a double-gap (3band) semiconductor.

Of course, these values are large compared with the maximum theoretical efficiencies of solar cells (dot curve labeled as 'REF' in figures 4 and 5). When all possible losses are considered (reflection, quantum-yield, absorption, collection, etc) the maximum realizable efficiency $\eta_{\text {real }}$ is the product of the energy storage efficiency $\eta_{A}$ and the efficiency from the rest of the system including the possible losses $\eta_{\text {other }}$, i.e. $\eta_{\text {real }}=\eta_{A} \eta_{\text {other }}$. The efficiency $\eta_{\text {real }}$ has been estimated to be about $10 \%$ for a single-gap and $16 \%$ for a double-junction system [17]. It corresponds to $\eta_{\text {other }} \sim 0.31-0.35$. As it has been previously mentioned multi-junction devices could supply sufficient voltage to drive water splitting. However, multijunction devices are costly, thus, single-junction devices would be preferred. The single-junction device can be made up with single- or double-gap semiconductors. The single-junction device with double-gap semiconductors has a larger efficiency than the double-junction device (approximately 49\% versus $46 \%$ ). However, as $\eta_{\text {other }}$ is very low, the efficiency gain $\Delta \eta_{\text {real }}$ of single-junction devices with double-gap semiconductors is only $0.3 \%$ larger than the double-junction device.

Note that the efficiency represented in figures 1 and 2 (constrained to $V_{0}$ with a efficiency $\eta=J V_{o} / P_{\text {inc }}$ ) is different from that of the figures 4 and 5 (constrained to $V_{o} \geqslant V_{\text {redox }}+V_{\text {lost }}$, and where the efficiency is the energy storage efficiency $\eta_{A}=J V_{\text {redox }} / P_{\text {inc }}$, different to $\eta$ ).

\subsection{Device with a fixed current}

Unlike the case where the photo-voltage is fixed to $V_{o}$, when the photo-current is fixed to $J_{o}$ the PVD can only be operated with a semiconductor having a small enough gap in order to provide the necessary photo-current. For this reason when the gap increases the efficiency tends to zero abruptly since the semiconductor is not able to supply the necessary current $J_{o}$. For high $J_{o}$, the range of gap values narrows towards lower values. Although this happens for all semiconductors, the narrowing of values is less accentuated for multi-gap semiconductors since they are able to supply more current than single-gap semiconductors. It can be observed by comparing figures 6 and 7 for single- and double-gap semiconductors respectively.
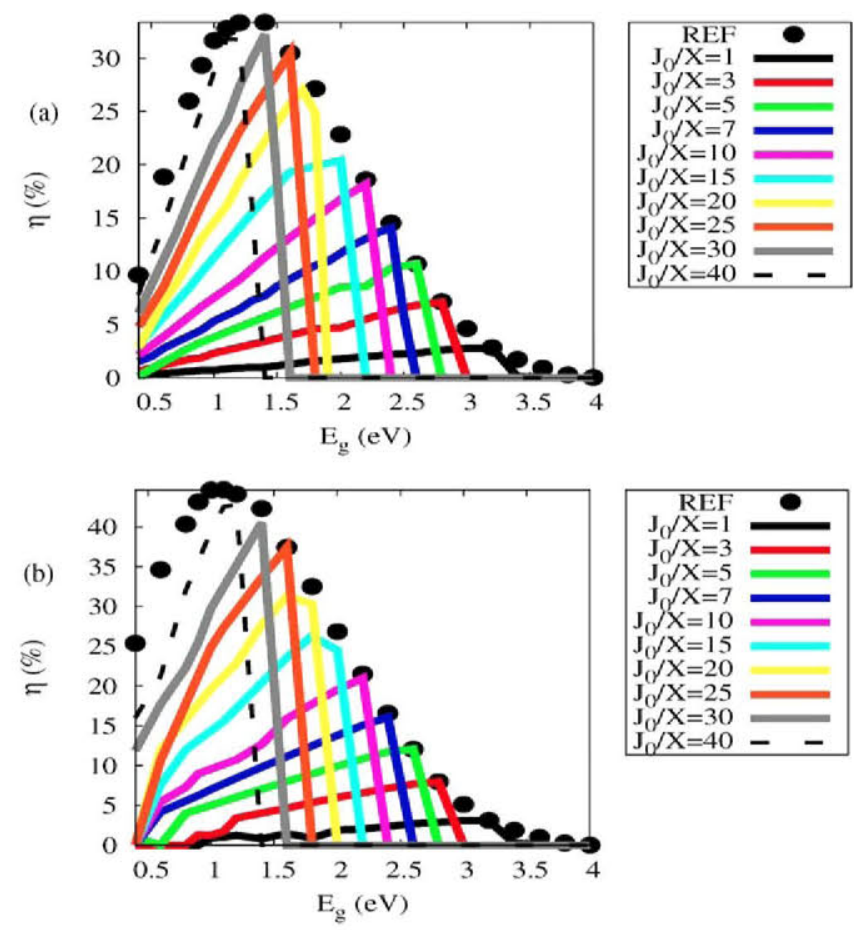

Figure 6. Same legend as that in figure 2, but for several output photocurrent $\mathrm{J}_{\mathrm{O}}\left(\mathrm{mA} \cdot \mathrm{cm}^{-3} / \mathrm{X}\right)$ values and a 2-band semiconductor.
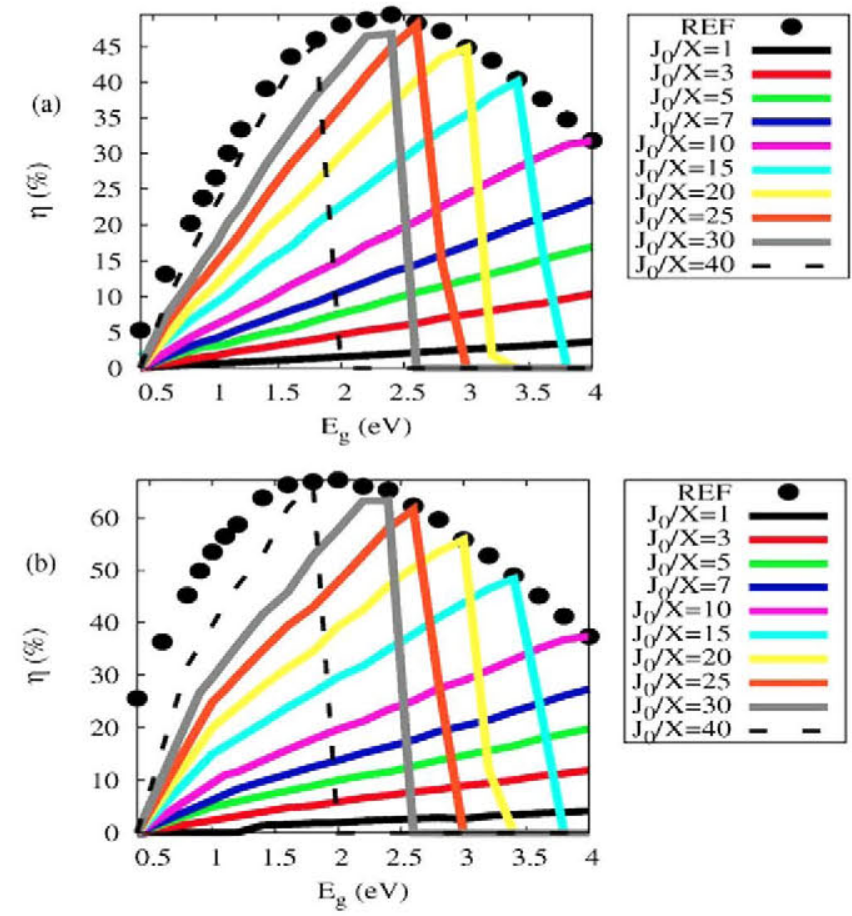

Figure 7. Same legend as that in figure 3 , but for several output photocurrent $\mathrm{J}_{\mathrm{O}}\left(\mathrm{mA} \cdot \mathrm{cm}^{-3} / \mathrm{X}\right)$ values and a 3-band semiconductor.

\section{Conclusions}

We have carried out a detailed analysis of the constrained maximum light to electric power conversion efficiencies when a PVD is used for generating a fixed voltage and/or 
current supplied to other devices. As absorbent materials of solar radiation we have considered the usual single-gap semiconductors and multi-gap semiconductors with intermediate bands between the usual valence and conduction bands. Both are evaluated. The radiative emission is considered and no approximations are made regarding the magnitude of the radiative emission with respect to the absorption. Our study is therefore not limited to non-degenerate semiconductors, i.e. the Fermi-Dirac and the Bose-Einstein statistics are not replaced by the Maxwell-Boltzmann statistics.

Although all semiconductors can be used, multi-gap semiconductors supply more current than conventional semiconductors and can be used with greater efficiency when coupled to electrochemical cells to store solar energy as chemical energy. Also, for this reason, they can supply a more constant current than single-gap semiconductors.

\section{Acknowledgments}

This work has been supported by the National Spanish projects MADRID-PV (S2013/MAE-2780, FEDER FUNDS) and INVENTA-PV (TEC2015-64189-C3-1-R, MINECO/FEDER).

\section{ORCID iDs}

César Tablero-Crespo (1) https://orcid.org/0000-00019721-1549

\section{References}

[1] Abdi F F and Berglund S P 2017 Recent developments in complex metal oxide photoelectrodes J. Phys. Appl. Phys. 50193002

[2] Sivula K 2016 Advanced device architectures and tandem devices Photoelectrochemical Solar Fuel Production: From
Basic Principles to Advanced Devices ed S Giménez and J Bisquert (Cham: Springer International Publishing) pp 493-512

[3] Archer M D and Nozik A J 2008 Nanostructured and Photoelectrochemical Systems for solar Photon Conversion (London: Imperial College Press)

[4] Memming R and Bahnemann D 2014 Semiconductor Electrochemistry (Weinheim, Bergstr: Wiley-VCH)

[5] Bolton J R, Haught A F and Ross R T 1981 Chapter 11photochemical energy storage: an analysis of limits Photochemical Conversion and Storage of Solar Energy ed J S Connolly (New York: Academic) pp 297-339

[6] Winkler M T, Cox C R, Nocera D G and Buonassisi T 2013 Modeling integrated photovoltaic-electrochemical devices using steady-state equivalent circuits Proc. Natl Acad. Sci. 110 E1076-82

[7] Luque A and Martí A 2003 Theoretical Limits of Photovoltaic Conversion, in Handbook of Photovoltaic Science and Engineering (New York: Wiley)

[8] Würfel P 2005 Physics of Solar Cells. From Principles to New Concepts (WILEY-VCH Verlag GmbH \& Co, KGaA)

[9] Green M A 2003 Third Generation Photovoltaics. Advanced Solar Energy Conversion (Berlin Heidelberg: Springer)

[10] Luque A and Martí A 1997 Increasing the efficiency of ideal solar cells by photon induced transitions at intermediate levels Phys. Rev. Lett. 78 5014-7

[11] Anon (http://rredc.nrel.gov/solar/spectra/am1.5/ ASTMG173/ASTMG173.html)

[12] Brown A S and Green M A 2002 Impurity photovoltaic effect: fundamental energy conversion efficiency limits $J$. Appl. Phys. 92 1329-36

[13] Tablero C 2015 Microscopic analysis and applications of the $\mathrm{Cu}(\mathrm{Sb}, \mathrm{Bi}) \mathrm{S} 2$ high optical absorption J. Phys. Chem. C 119 $8857-63$

[14] Tablero C 2015 Optical absorption analysis of quaternary molybdate- and tungstate-ordered double perovskites J. Alloys Compd. $639203-9$

[15] Crespo C T 2018 Potentiality of CuFeO2-delafossite as a solar energy converter Sol. Energy $163162-6$

[16] Tablero C 2015 Optical absorption and applications of the $\mathrm{ABO} 4(\mathrm{~A}=\mathrm{Ca}, \mathrm{Pb}$ and $\mathrm{B}=\mathrm{Mo}, \mathrm{W})$ semiconductors Chem. Phys. Lett. 635 190-5

[17] Bolton J R, Strickler S J and Connolly J S 1985 Limiting and realizable efficiencies of solar photolysis of water Nature 316495 